\title{
Extração de compostos bioativos da polpa de jambo com diferentes tipos de solventes
}

\author{
Extraction of bioactive compounds from jambo pulp with different types of solvents \\ Extracción de compuestos bioactivos de pulpa de jambo con diferentes tipos de solventes
}

Recebido: 23/07/2021 | Revisado: 01/08/2021 | Aceito: 05/08/2021 | Publicado: 10/08/2021

\author{
Nkarthe Guerra Araújo \\ ORCID: https://orcid.org/0000-0002-6506-051X \\ Universidade Federal da Paraíba, Brasil \\ E-mail: nkarthe@gmail.com \\ Lucivânia Assis de Oliveira Navarro \\ ORCID: https://orcid.org/0000-0001-5834-3805 \\ Universidade Federal da Paraíba, Brasil \\ E-mail: lucivania_nf@ hotmail.com \\ Cláudia Souza Macêdo \\ ORCID:https://orcid.org/0000-0002-3173-1821 \\ Universidade Federal do Rio Grande do Norte, Brasil \\ E-mail:csmacedo.ufrn@gmail.com \\ Haíssa Roberta Cardarelli \\ ORCID:https://orcid.org/0000-0002-8087-3017 \\ Universidade Federal da Paraíba, Brasil \\ E-mail:hrcarda@gmail.com
}

\begin{abstract}
Resumo
Este trabalho teve por objetivo definir o melhor solvente extrator dos compostos bioativos da polpa de jambo, armazenada pelos métodos de congelamento e liofilização. Para tanto, frutos de Syzygium malaccensis, provenientes do Estado do Rio Grande do Norte-RN foram processados, as polpas obtidas foram congeladas ou liofilizadas e também caracterizadas físico-quimicamente, enquanto seus teores de compostos bioativos foram determinados. Verificou-se aumento significativo nos teores de proteínas, lipídeos, cinzas, açúcares redutores, sólidos solúveis, ácido ascórbico e carotenoides totais da polpa liofilizada quando comparada à congelada. A polpa liofilizada apresentou ainda, atividade antioxidante significativamente superior $(24,19 \mu \mathrm{mol} \mathrm{TE} \mathrm{g} \pm 0,09)$ à congelada $\left(17,10 \mu \mathrm{mol} \mathrm{TE} \mathrm{g}{ }^{-1} \pm\right.$ $0,56)$ e resultados satisfatórios de solubilidade $(67,03 \%)$ e higroscopicidade $(9,38 \%)$. Os teores de flavonoides amarelos, antocianinas monoméricas totais e de compostos fenólicos totais variaram conforme o tipo de solvente utilizado e a proporção de suas misturas. O metanol-HCl 1,5 M foi o solvente mais eficaz na extração de compostos fenólicos totais e de antocianinas, enquanto a água pura destacou-se na extração de flavonoides amarelos. Ambos os processos indicaram que o jambo é fonte de compostos bioativos, todavia, a liofilização preservou melhor a estabilidade dos compostos estudados, sugerindo-se esse método como o mais apropriado para obtenção da polpa de jambo, a fim de preservar melhor o potencial funcional deste fruto.
\end{abstract}

Palavras-chave: Ácido ascórbico; Antocianinas; Compostos fenólicos totais.

\begin{abstract}
This work aimed to define the best solvent extracting bioactive compounds from the jambo pulp, stored by the methods of freezing and lyophilization. For this purpose, Syzygium malaccensis fruits from the State of Rio Grande do Norte-RN were processed, the pulps obtained were frozen or lyophilized and also physicochemically characterized, while their contents of bioactive compounds were determined. There was a significant increase in the contents of proteins, lipids, ash, reducing sugars, soluble solids, ascorbic acid and total carotenoids of the lyophilized pulp when compared to the frozen one. The lyophilized pulp also presented significantly higher antioxidant activity $(24.19 \mu \mathrm{mol}$ $\mathrm{TE} \mathrm{g} \pm 0.09)$ than the frozen one $\left(17.10 \mu \mathrm{mol} \mathrm{TE} \mathrm{g}^{-1} \pm 0.56\right)$ and satisfactory solubility results $(67.03 \%)$ and hygroscopicity $(9.38 \%)$. The contents of yellow flavonoids, total monomeric anthocyanins and total phenolic compounds varied according to the type of solvent used and the proportion of their mixtures. $1.5 \mathrm{M}$ methanol-HCl was the most effective solvent for extracting total phenolic compounds and anthocyanins, while pure water stood out for extracting yellow flavonoids. Both processes indicated that jambo is a source of bioactive compounds, however, lyophilization better preserved the stability of the studied compounds, suggesting this method as the most appropriate for obtaining jambo pulp, in order to better preserve its functional potential fruit.
\end{abstract}

Keywords: Ascorbic acid; Anthocyanins; Total phenolic compounds.

\section{Resumen}

Este trabajo tuvo como objetivo definir el mejor solvente extrayendo compuestos bioactivos de la pulpa de jambo, almacenada por los métodos de congelación y liofilización. Para ello se procesaron frutos de Syzygium malaccensis 
del Estado de Rio Grande do Norte-RN, se congelaron o liofilizaron las pulpas obtenidas y también se caracterizaron fisicoquímicamente, mientras se determinaba su contenido en compuestos bioactivos. Hubo un aumento significativo $(\mathrm{p}<0.05)$ en el contenido de proteínas, lípidos, cenizas, azúcares reductores, sólidos solubles, ácido ascórbico y carotenoides totales de la pulpa liofilizada en comparación con la congelada. La pulpa liofilizada también presentó actividad antioxidante significativamente mayor $(24,19 \mu \mathrm{mol}$ TE g $\pm 0,09)$ que la congelada $\left(17,10 \mu \mathrm{mol} \mathrm{TE} \mathrm{g}^{-1} \pm\right.$ $0,56)$ y resultados satisfactorios de solubilidad $(67,03 \%)$ e higroscopicidad $(9,38 \%)$. Los contenidos de flavonoides amarillos, antocianinas monoméricas totales y compuestos fenólicos totales variaron según el tipo de disolvente utilizado y la proporción de sus mezclas. El metanol- $\mathrm{HCl}$ 1,5 M fue el solvente más efectivo para extraer compuestos fenólicos totales y antocianinas, mientras que el agua pura se destacó por extraer flavonoides amarillos. Ambos procesos indicaron que el jambo es fuente de compuestos bioactivos, sin embargo, la liofilización preservó mejor la estabilidad de los compuestos estudiados, sugiriendo este método como el más apropiado para la obtención de pulpa de jambo, con el fin de preservar mejor su potencial funcional.

Palabras clave: Ácido ascórbico; Antocianinas; Compuestos fenólicos totales.

\section{Introdução}

Diversas pesquisas têm evidenciado o potencial antioxidante de uma variedade de frutas. O consumo frequente destas tem sido associado à baixa incidência de doenças degenerativas, tais como câncer, doenças cardíacas, inflamatórias e do sistema imunológico, além de disfunções neurológicas. Essas evidências estão relacionadas com a presença de compostos antioxidantes variados presentes nas mesmas (Milani, et al., 2012; Kibe, et al., 2017; Rocha, et al., 2021).

O jambeiro vermelho (Syzygium malaccensis) é uma árvore frutífera pertencente à família Myrtaceae, da qual diferentes partes da planta são utilizadas para consumo e fins medicinais (Li, et al., 2015). A facilidade de cultivo e condições climáticas favoráveis permitiu uma expansão significativa de sua produção no Brasil, principalmente nas regiões Sudeste, Norte e Nordeste (Nunes, et al., 2016). No entanto, seu fruto é utilizado para consumo direto apenas nas regiões produtoras, o que resulta num grande desperdício na época da safra devido à reduzida vida útil dos frutos e escassez de informações relacionadas à viabilidade tecnológica para sua industrialização (Augusta, et al., 2010). Neste sentido, a produção de polpa a partir do fruto é alternativa para o seu aproveitamento na época da safra, permitindo a oferta também nos períodos de entressafra, evitando problemas relacionados à sazonalidade.

Estudos referentes à caracterização do jambo vermelho das regiões Sudeste e Nordeste do Brasil realizados por Batista et al. (2016) e Nunes et al. (2016), reportaram variações nos teores de antocianinas de 12,90 e 1045 mg cianidina-3glicosídeo $100 \mathrm{~g}^{-1}$, compostos fenólicos totais de 14,81 e $1293 \mathrm{mg}$ EAG $100 \mathrm{~g}^{-1}$, e na atividade antioxidante de 674.75 e 25,92 $\mu \mathrm{mol} \mathrm{TEg}^{-1}$, respectivamente. Entretanto, pesquisas referentes à caracterização da polpa de jambo vermelho oriundo do Estado do Rio Grande do Norte (RN) e de seu conteúdo de compostos bioativos antioxidantes são escassas.

Um dos fatores mais importantes que afetam a extração de compostos bioativos de matrizes vegetais é o solvente de extração utilizado (Van Ngo, et al., 2017), dado que a solubilidade dos compostos bioativos em um solvente específico é uma característica peculiar dos fitoquímicos presentes na matriz avaliada, o que justifica a inexistência de um procedimento universal para sua determinação (Santos \& Gonçalves, 2016). Tradicionalmente, a extração de polifenóis é realizada usando misturas de solventes orgânicos voláteis tais como metanol, etanol, acetona, acetonitrila e suas combinações, podendo incluir diferentes proporções de água (Rajbhar, et al., 2015). Entretanto, não há relatos na literatura referentes à definição do melhor solvente extrator de compostos bioativos da polpa de jambo vermelho, sendo este, o primeiro estudo com esta finalidade.

Processos de desidratação que não empregam o calor como mecanismo de remoção da água, como a liofilização, são eficientes para minimizar a degradação dos compostos termolábeis dos alimentos (Silva-Espinoza, et al., 2019). A liofilização apresenta também outras vantagens como redução de custos com embalagem, armazenamento e transporte, abrangendo tanto a indústria alimentícia como farmacêutica (Oyinloye \& Yoon, 2020). Todavia, dentre as alternativas de conservação para polpa de fruta, o congelamento ainda é o procedimento mais utilizado (Carvalho, et al., 2017). 
Assim, este trabalho teve por objetivo definir o melhor solvente extrator dos compostos bioativos antioxidantes da polpa de jambo armazenada pelos métodos de congelamento e liofilização.

\section{Metodologia}

Essa pesquisa é de natureza quantitativa, conforme descrito por Pereira et al. (2018). O jambo vermelho $(42,5 \mathrm{~kg})$ foi adquirido na Unidade Acadêmica Especializada em Ciências Agrárias da Universidade Federal do Rio Grande do Norte, Campus de Macaíba-RN. Os frutos foram colhidos manualmente em estágio fisiológico de maturação completo considerando a coloração da casca vermelha intensa, foram higienizados em água corrente, sanitizados em água clorada a 100 ppm por 15 min, secos a $28 \pm 1^{\circ} \mathrm{C}$, e pesados. Posteriormente sofreram corte longitudinal para remoção da semente, foram fracionados com casca (forma aproximada de retângulos de aproximadamente $4,5 \mathrm{~cm}$ ) e despolpados em despolpadeira de frutas semi-industrial Modelo 0,25 dF (Itametal, Itabuna, Bahia, Brasil) com peneira de 1,5 mm de diâmetro. A polpa obtida (30,0 $\mathrm{kg})$ foi pesada, dividida em duas porções, uma sendo encaminhada para liofilização, e outra acondicionada em embalagens de nylonpolietileno de $150 \mathrm{~g}$ e submetida ao congelamento lento em freezer horizontal (Eletrolux, São Paulo, Brasil) a $-18{ }^{\circ} \mathrm{C}$ para análises posteriores.

A polpa liofilizada foi obtida dispondo-se $100 \mathrm{~g}$ da polpa de jambo fresca em bandejas de aço em inox de $18 \mathrm{~cm}$ e acondicionando a $-18{ }^{\circ} \mathrm{C} / 48 \mathrm{~h}$. Após congelamento, as bandejas foram transferidas para o liofilizador Modelo LT 1500 (Terroni, São Carlos, São Paulo, Brasil). As condições de operação foram: temperatura de $-30^{\circ} \mathrm{C}$ por tempo de $48 \mathrm{~h}$, velocidade constante de $1 \mathrm{~mm} / \mathrm{h}$, vácuo variando de $80 \mu \mathrm{Hg}$ até $200 \mu \mathrm{Hg}$. Os pós obtidos foram acondicionados em embalagens de nylonpolietileno, selados a vácuo, e em seguida acondicionados em embalagens metalizadas, onde foram armazenados em temperatura ambiente $\left(28 \pm 1^{\circ} \mathrm{C}\right)$ ao abrigo da luz.

\subsection{Caracterização das polpas}

As polpas foram submetidas às análises de acidez titulável (AOAC 942.15), sólidos solúveis totais (AOAC 932.12), pH (AOAC 947.05), açúcares redutores (AOAC 923.09), umidade (AOAC 934.06), cinzas (AOAC 940.26), teores de lipídeos (AOAC 920.39), proteínas (AOAC 960.52) e de ácido ascórbico (AOAC 967.21) (AOAC, 2000). A análise de atividade de água foi realizada em medidor AQUALAB modelo CX2 (Decagon Devices, Washington, EUA) segundo as recomendações do fabricante. Análises de solubilidade (Cano-Chauca, et al., 2005) e higroscopicidade (López-Córdoba, et al., 2014) foram realizadas apenas na polpa liofilizada.

As análises de compostos fenólicos totais (Obanda et al., 1997), flavonoides amarelos (Francis, 1982) e antocianinas monoméricas totais (Giusti \& Wrolstad, 2001) foram realizadas após a obtenção dos extratos com diferentes tipos de solventes: água destilada, metanol e etanol PA (Neon, São Paulo, Brasil) e ácido clorídrico PA (Vetec, Duque de Caxias, Rio de Janeiro, Brasil), enquanto os carotenóides totais foram extraídos com acetona PA (Lichtenthaler \& Buschmann, 2001) e a atividade antioxidante (Brand-Williams, et al., 1995) nos extratos hidroetanólicos. Todas as análises foram realizadas em triplicata.

\subsection{Avaliação dos compostos bioativos e atividade antioxidante}

\subsubsection{Obtenção dos extratos}

A determinação de compostos fenólicos totais, flavonoides amarelos e de antocianinas monoméricas totais foi realizada com base em testes preliminares, empregando-se 5,0g e 1,5g, respectivamente da polpa de jambo congelada e liofilizada, macerando-as com $25 \mathrm{~mL}$ dos diferentes tipos de solventes: água destilada; metanol e etanol (99,9\% v/v); etanol/HCl; metanol/HCl e água destilada/HCl (85:15 v/v) (Francis, 1982), metanol/água destilada e etanol/água destilada (70:30 v/v) (Borges, et al., 2011). Após maceração, as amostras foram transferidas para balões de $50 \mathrm{~mL}$ e seus volumes foram 
ajustados com os respectivos solventes. Transferiu-se para tubos de centrífuga do tipo Falcon onde permaneceram ao abrigo da luz a $8 \pm 2{ }^{\circ} \mathrm{C}$ por $24 \mathrm{~h}$. Transcorrido o período, os extratos foram filtrados em papel de filtro qualitativo e analisados posteriormente.

\subsubsection{Determinação de compostos fenólicos totais, flavonoides amarelos, carotenoides totais e antocianinas monoméricas} totais

O conteúdo de compostos fenólicos totais foi quantificado conforme descrito por Obanda et al. (1997). Para tanto, alíquotas de $0,5 \mathrm{~mL}$ e de $0,3 \mathrm{~mL}$ dos extratos das polpas congelada e liofilizada respectivamente, foram transferidas para tubos de ensaio, aos quais foram adicionados $1 \mathrm{~mL}$ do reagente Folin-Ciocalteau 1:3N (Sigma-Aldrich, São Paulo, Brasil), $2 \mathrm{~mL}$ de carbonato de sódio a $20 \%$ (m/v) (Sigma-Aldrich, São Paulo, Brasil) e $2 \mathrm{~mL}$ de água destilada. Após reação por $30 \mathrm{~min} \mathrm{em}$ câmara escura a $28 \pm 1{ }^{\circ} \mathrm{C}$, as absorbâncias foram medidas em espectrofotômetro digital Modelo $722 \mathrm{~N}$ (Edutec, São Paulo, Brasil) no comprimento de onda de $700 \mathrm{~nm}$. Os resultados foram expressos em miligramas de equivalentes de ácido gálico por cem gramas da amostra (mg EAG $\left.100 \mathrm{~g}^{-1}\right)$.

A análise de flavonoides amarelos foi realizada segundo metodologia proposta por Francis (1982), na qual a absorbância dos extratos foi mensurada a $374 \mathrm{~nm}$. Já os carotenoides totais foram extraídos misturando-se $5,0 \mathrm{~g}$ das respectivas polpas com $25 \mathrm{~mL}$ de acetona PA (Anidrol, Diadema, São Paulo, Brasil). Os extratos obtidos foram imediatamente filtrados em papel de filtro qualitativo e analisados em espectrofotômetro Modelo 722N (Edutec, São Paulo, Brasil) nos comprimentos de onda de 470, 646 e $663 \mathrm{~nm}$ (Lichtenthaler \& Buschmann, 2001). Os resultados de ambos os compostos foram expressos em

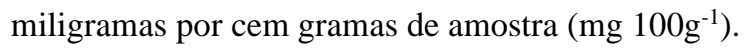

$\mathrm{O}$ conteúdo de antocianinas monoméricas totais (AMT) foi determinado por diferença de $\mathrm{pH}$, conforme descrito por Giusti; Wrolstad (2001), no qual foram adicionados $1,6 \mathrm{~mL}$ das soluções tampão de cloreto de potássio $0,025 \mathrm{M}(\mathrm{pH}=1)$ (Sigma-Aldrich, São Paulo, Brasil), ou acetato de sódio 0,4 M (pH= 4,5) (Synth, Diadema, São Paulo, Brasil) a 0,4 mL do extrato. As amostras foram homogeneizadas e mantidas na ausência da luz a $28 \pm 1{ }^{\circ} \mathrm{C}$ por $30 \mathrm{~min}$. A leitura foi realizada em espectrofotômetro digital Modelo 722N (Edutec, São Paulo, Brasil) na faixa de $510 \mathrm{~nm}$ e a $700 \mathrm{~nm}$. O conteúdo de AMT foi calculado conforme Equação 1.

$\mathrm{AMT}=\mathrm{A} \times \mathrm{MW} \times 1000 /(\varepsilon \times \mathrm{C})$

Sendo:

A: Absorbância (A510nm - A700nm) pH 1,0 - (A510nm - A700nm) pH 4,5;

MW: massa molecular da cianidina-3-glicosídeo $=490 \mathrm{~g} \mathrm{~mol}^{-1}$;

$\varepsilon$ : Absortividade molar de cianidina-3-glicosídeo $=26900 \mathrm{M}^{-1} \mathrm{~cm}^{-1}$;

C: Concentração do tampão $\left(\mathrm{mg} \mathrm{mL}^{-1}\right)$

A atividade antioxidante foi determinada através da capacidade dos antioxidantes presentes nas amostras em desativar o radical estável DPPH•, segundo metodologia adaptada de Brand-Williams et al. (1995). Para tanto, utilizou-se em substituição à solução metanólica, solução etanólica de DPPH (Sigma-Aldrich, São Paulo, Brasil) e as determinações foram realizadas em microplacas de poliestireno com 96 poços (TPP, Suíça), para onde foram adicionados $40 \mu \mathrm{L}$ de extrato preparado com etanol:água destilada (70:30 v/v) e $200 \mu \mathrm{L}$ da solução de DPPH. As leituras das absorbâncias foram realizadas após 60 min de reação no escuro a $28 \pm 1^{\circ} \mathrm{C}$, em espectrofotômetro Modelo Cary 50 (Walnut Creeck, USA) a $515 \mathrm{~nm}$. A 
capacidade antioxidante das amostras foi calculada em relação à atividade do antioxidante sintético Trolox (Sigma-Aldrich, São Paulo, Brasil).

\subsection{Delineamento experimental e análise estatística}

Os experimentos foram realizados empregando o delineamento inteiramente casualizado (DIC) e os resultados analisados por análise de variância (ANOVA), com comparação das médias dos tipos de solventes utilizados pelo teste de Tukey ao nível de 5\% de significância, utilizando-se o software Statistica versão 7.0. A comparação das médias dos dois tipos de polpa distintos utilizou o teste t-Student, ao nível de 5\% de significância.

\section{Resultados e Discussão}

\subsection{Caracterização físico-química das polpas}

A Tabela 1 apresenta os resultados da caracterização físico-química da polpa de jambo submetida aos dois processos de conservação distintos. Verificou-se aumento significativo $(\mathrm{p}<0,05)$ nos teores de proteínas, lipídeos, cinzas, açúcares redutores, sólidos solúveis, ácido ascórbico e carotenoides totais da polpa liofilizada quando comparada à congelada. Do mesmo modo, foi observada redução significativa nos parâmetros, atividade de água e umidade. A redução destes é, contudo, desejável e uma característica típica de produtos obtidos por processos de desidratação (liofilização), enquanto o aumento nos teores de ácido ascórbico e carotenoides totais comprova a eficiência deste processo na retenção dos nutrientes.

Tabela 1 - Composição centesimal das polpas de jambo congelada e liofilizada expressa em percentual $\left(\mathrm{mg}^{100 \mathrm{~g}^{-1}}\right)$.

\begin{tabular}{lcc}
\hline \multicolumn{1}{c}{ Parâmetros } & Polpa de jambo congelada & Polpa de jambo liofilizada \\
\hline Umidade & $91,09^{\mathrm{a}} \pm 0,32$ & $8,75^{\mathrm{b}} \pm 0,24$ \\
Proteínas & $0,71^{\mathrm{b}} \pm 0,03$ & $2,02^{\mathrm{a}} \pm 0,11$ \\
Lipídeos & $0,45^{\mathrm{b}} \pm 0,39$ & $1,98^{\mathrm{a}} \pm 0,41$ \\
Cinzas & $0,23^{\mathrm{b}} \pm 0,07$ & $4,60^{\mathrm{a}} \pm 0,15$ \\
Açúcares redutores & $3,21^{\mathrm{b}} \pm 0,25$ & $42,53^{\mathrm{a}} \pm 4,69$ \\
Sólidos solúveis & $7,70^{\mathrm{b}} \pm 0,00$ & $80,00^{\mathrm{a}} \pm 0,00$ \\
pH & $3,36^{\mathrm{b}} \pm 0,15$ & $3,67^{\mathrm{a}} \pm 0,19$ \\
Atividade de água & $0,941^{\mathrm{a}} \pm 0,00$ & $0,259^{\mathrm{b}} \pm 0,00$ \\
Ácido ascórbico & $118,00^{\mathrm{b}} \pm 0,00$ & $161,90^{\mathrm{a}} \pm 0,42$ \\
Carotenoides totais & $42,30^{\mathrm{b}} \pm 0,86$ & $44,99^{\mathrm{a}} \pm 1,55$ \\
Solubilidade & $\mathrm{ND}$ & $67,03 \pm 0,51$ \\
Higroscopicidade & $\mathrm{ND}$ & $9,38 \pm 0,30$ \\
\hline
\end{tabular}

Letras diferentes na mesma linha indica diferença estatisticamente significativa pelo teste t-Student $(\mathrm{p}<0,05)$. ND indica que o parâmetro não foi determinado. Fonte: Autores.

Os teores de proteínas, lipídeos e açúcares redutores da polpa liofilizada foram inferiores aos valores relatados por Batista et al. (2016), para os mesmos parâmetros: 5,33\%, 2,62\% e 49,55\%, respectivamente, no mesmo tipo de polpa, oriunda de frutos de jambeiro provenientes do Sudeste do Brasil. Já o teor de umidade, lipídeos, açúcares redutores e sólidos solúveis da polpa congelada foram superiores, e o teor proteico, de cinzas e o $\mathrm{pH}$ ligeiramente inferiores aos resultados obtidos por Nunes et al. (2016), em análise da composição de frutos de jambo vermelho provenientes da cidade de Sapé-PB, indicando, portanto, diferenças também, na composição dos frutos da mesma região.

Conforme apresentado na Tabela 1, a polpa de jambo liofilizada apresentou teor elevado de solubilidade e baixo teor de higroscopicidade. Estes resultados são considerados satisfatórios tendo em vista que durante o processo de liofilização não foi adicionado nenhum adjuvante de secagem a polpa. Krumreich et al. (2016) avaliando polpa de ubaia liofilizada com adição de maltodextrina, obtiveram resultados inferiores de higroscopicidade (4,58\%) e de solubilidade (49,79\%). Estudos que 
avaliaram os mesmos parâmetros em polpas desidratadas de Syzygium malaccensis, entretanto, não foram encontrados durante esta pesquisa.

A polpa congelada apresentou resultado inferior no conteúdo de ácido ascórbico em relação à liofilizada $(\mathrm{p}<0,05)$. Todavia, os teores de ácido ascórbico para ambas foram elevados, indicando que independentemente do método de obtenção da polpa, o jambo vermelho destaca-se como fonte desta vitamina. Conteúdo inferior foi relatado por Shajib et al. (2013) para o Syzygium cumini (25,7 mg 100 $\mathrm{g}^{-1}$ ) fresco e por Gordon et al. (2011), para o jambolão liofilizado (93,5 mg $\left.100 \mathrm{~g}^{-1}\right)$.

O teor de carotenoides totais obtidos a partir da extração com acetona PA foi superior aos achados de Batista et al. (2016), que avaliando o mesmo composto a partir da extração com isopropanol e hexano obtiveram $0,046 \mathrm{mg}^{100 \mathrm{~g}^{-1} \mathrm{de}}$ carotenoides totais para a polpa de jambo obtida no Sudeste do Brasil. Fatores como a existência de vários tipos de carotenoides e a variação nas quantidades de carotenoides dentro de um mesmo alimento, a distribuição não uniforme deste composto entre amostras, a natureza variável das matrizes alimentícias, dentre outros, são fatores que afetam sua determinação (Nellis, et al., 2017), o que poderia explicar a variação entre os resultados obtidos para o mesmo tipo de fruto proveniente de regiões distintas.

\subsection{Extração de compostos bioativos com diferentes tipos de solventes, sua determinação e avaliação da atividade antioxidante}

Os resultados mostraram que os teores de compostos fenólicos totais, flavonoides amarelos e antocianinas monoméricas totais variaram significativamente $(\mathrm{p}<0,05)$ entre a polpa congelada e a liofilizada e conforme o tipo de solvente utilizado e a proporção de suas misturas ( $\mathrm{p}<0,05)$ (Tabelas 2,3 e 4).

O conteúdo total de compostos fenólicos (Tabela 2), diferiu significativamente entre a polpa congelada e liofilizada e corroboram os achados de Batista et al. (2016) em extratos hidrometanólicos (20:80 v/v) de polpa de jambo submetida ao mesmo método de obtenção.

Tabela 2 - Conteúdo total de compostos fenólicos em polpa de jambo obtidos a partir de diferentes solventes.

\begin{tabular}{lcc}
\hline Solventes & $\begin{array}{c}\text { Polpa congelada mg } \\
\text { EAG 100g-1 }\end{array}$ & $\begin{array}{c}\text { Polpa liofilizada mg } \\
\text { EAG 100g }^{-1}\end{array}$ \\
\hline Água & $34,60^{\mathrm{Bb}} \pm 0,13$ & $370,28^{\mathrm{Ab}} \pm 0,63$ \\
Metanol/HCl 1,5N (85:15 v/v) & $35,18^{\mathrm{Ba}} \pm 0,11$ & $388,93^{\mathrm{Aa}} \pm 1,25$ \\
Etanol/HCl 1,5N (85: 15 v/v) & $34,11^{\mathrm{Bb}} \pm 0,07$ & $300,68^{\mathrm{Ac}} \pm 1,26$ \\
Água/ HCl 1,5N (85:15 v/v) & $27,01^{\mathrm{Bd}} \pm 0,06$ & $249,60^{\mathrm{Af}} \pm 1,13$ \\
Etanol/água (70:30 v/v) & $34,05^{\mathrm{Bb}} \pm 0,57$ & $262,71^{\mathrm{Ae}} \pm 0,31$ \\
Metanol/água (70:30 v/v) & $25,59^{\mathrm{Be}} \pm 0,41$ & $292,09^{\mathrm{Ad}} \pm 0,83$ \\
Etanol (99,9\%) & $33,01^{\mathrm{Bc}} \pm 0,19$ & $160,67^{\mathrm{Ah}} \pm 0,83$ \\
Metanol (99,9\%) & $32,95^{\mathrm{Bc}} \pm 0,52$ & $231,18^{\mathrm{Ag}} \pm 2,50$ \\
\hline
\end{tabular}

Letras maiúsculas diferentes na mesma linha indicam diferença significativa entre os tipos de polpa pelo teste tStudent $(\mathrm{p}<0,05)$. Letras minúsculas na mesma coluna indicam diferença estatisticamente significativa entre os tipos de solvente, pelo teste de Tukey $(\mathrm{p}<0,05)$. Fonte: Autores. 
Conforme observado na Tabela 2, o metanol acidificado foi o solvente mais eficiente na extração de compostos fenólicos totais, para ambas as polpas, seguido pelos resultados obtidos a partir da extração com água pura e etanol acidificado. O emprego da água como solvente parece mais indicado, pois além da eficiência na extração, quando comparada ao metanol e etanol PA, apresenta vantagens por evitar riscos de exposição do analista a reagentes tóxicos e reduz gastos com reagentes químicos. Moura Filho et al. (2017) também obtiveram maior eficiência na extração de compostos fenólicos em polpa de buriti usando água pura, quando comparado à extração com metanol e etanol PA. Estudos referentes à determinação de polifenois, flavonoides e antocianinas em polpa de jambo utilizando a água pura como solvente de extração até o momento não foram reportados na literatura.

A extração de fenólicos com etanol puro de ambas as polpas foi significativamente inferior $(p<0,05)$ quando comparado aos resultados dos extratos hidroetanólicos, todavia, a polpa de jambo congelada apresentou teor significativamente superior de compostos fenólicos com metanol puro $(\mathrm{p}<0,05)$ em comparação ao extrato hidrometanólico. A solubilidade dos compostos fenólicos em um determinado solvente está relacionada com a composição química da matriz do alimento de onde são extraídos, apontando para a necessidade de seleção criteriosa do método de extração (Milani, et al., 2012).

Os teores de flavonoides amarelos da polpa congelada foram baixos e significativamente inferiores à liofilizada ( $\mathrm{p}<$ 0,05) (Tabela 3). Entretanto, esses resultados são satisfatórios quando comparado aos achados de Silva et al. (2014) que investigando o conteúdo de flavonoides amarelos em diferentes frutos tropicais (polpas de abacaxi, acerola, caju, goiaba, graviola, mamão e manga), não constataram a presença desse fitoquímico na composição.

Tabela 3 - Conteúdo de flavonoides amarelos em polpa de jambo obtidos a partir de diferentes solventes.

\begin{tabular}{|c|c|c|}
\hline Solventes & Polpa congelada mg $100 \mathrm{~g}^{-1}$ & Polpa liofilizada mg $100 \mathrm{~g}^{-1}$ \\
\hline Água & $3,60^{\mathrm{Ba}} \pm 0,02$ & $34,86^{\mathrm{Aa}} \pm 0,03$ \\
\hline Metanol/HCl 1,5N (85:15 v/v) & $2,82^{\mathrm{Bb}} \pm 0,03$ & $24,15^{\mathrm{Ab}} \pm 0,18$ \\
\hline Etanol/HCl 1,5N (85: $15 \mathrm{v} / \mathrm{v})$ & $2,84^{\mathrm{Bb}} \pm 0,01$ & $22,26^{\mathrm{Ac}} \pm 0,15$ \\
\hline Água/ $\mathrm{HCl} 1,5 \mathrm{~N}(85: 15 \mathrm{v} / \mathrm{v})$ & $2,57^{\mathrm{Bc}} \pm 0,02$ & $20,04^{\mathrm{Ad}} \pm 0,09$ \\
\hline Etanol/água $(70: 30 \mathrm{v} / \mathrm{v})$ & $2,13^{\mathrm{Bd}} \pm 0,02$ & $15,88^{\mathrm{Ae}} \pm 0,07$ \\
\hline Metanol/água (70:30 v/v) & ND & $13,49^{\mathrm{f}} \pm 0,06$ \\
\hline Etanol $(99,9 \%)$ & $1,73^{\mathrm{Be}} \pm 0,01$ & $9,24^{\mathrm{Ah}} \pm 0,08$ \\
\hline Metanol $(99,9 \%)$ & ND & $11,04^{\mathrm{g}} \pm 0,17$ \\
\hline
\end{tabular}

Letras maiúsculas diferentes na mesma linha indicam diferença significativa entre os tipos de polpa pelo teste t-Student $(\mathrm{p}<0,05)$. Letras minúsculas na mesma coluna indicam diferença estatisticamente significativa entre os tipos de solvente, pelo teste de Tukey ( $\mathrm{p}<0,05)$. ND indica leituras abaixo de 0,20 nm (resultado não detectado). Fonte: Autores.

A água pura destacou-se como o melhor solvente extrator de flavonoides amarelos na polpa congelada e liofilizada, seguido por metanol/ $\mathrm{HCl}$ e etanol/ $\mathrm{HCl}$ (Tabela 3). Os teores de flavonoides amarelos obtidos utilizando-se solventes com polaridades distintas para a polpa congelada foram inferiores aos valores reportados por Batista et al. (2016), utilizando o metanol (80\%) como solvente extrator para a polpa de jambo vermelho fresca $\left(12,86 \mathrm{mg}^{100 \mathrm{~g}^{-1}}\right)$, proveniente da região Sudeste do Brasil. 
O teor de antocianinas monoméricas totais da polpa congelada variou de $10,35 \pm 0,05$ a $18,44 \pm 0,06 \mathrm{mg}$ cianidina-3glicosídeo $100 \mathrm{~g}^{-1}$ enquanto para a polpa liofilizada a variação foi de 41,69 $\pm 0,23$ a 138,83 $\pm 0,41 \mathrm{mg}$ cianidina-3-glicosídeo $100 \mathrm{~g}^{-1}$ (Tabela 4). Esses resultados aproximam-se dos achados de Batista et al. (2016), para a polpa de jambo fresca (12,90 mg cianidina-3-glicosídeo $\left.100 \mathrm{~g}^{-1}\right)$ e liofilizada (126,95 mg cianidina-3-glicosídeo $\left.100 \mathrm{~g}^{-1}\right)$ proveniente da cidade de Campinas-SP.

Tabela 4 -Conteúdo total de antocianinas monoméricas em extratos de polpa de jambo obtidas a partir de diferentes solventes.

\begin{tabular}{lcc}
\hline Solventes & $\begin{array}{c}\text { Polpa congelada } \\
\text { mg cianidina-3 glicosídeo } \\
\mathbf{1 0 0 \mathbf { g } ^ { - 1 }}\end{array}$ & $\begin{array}{c}\text { Polpa liofilizada } \\
\text { mg cianidina -3-glicosídeo } \\
\mathbf{1 0 0 \mathbf { g } ^ { - 1 }}\end{array}$ \\
\hline Água & $10,35^{\mathrm{Bg}} \pm 0,05$ & $57,68^{\mathrm{Af}} \pm 0,20$ \\
Metanol/HCl 1,5N (85:15 v/v $)$ & $18,44^{\mathrm{Ba}} \pm 0,05$ & $138,83^{\mathrm{Aa}} \pm 0,41$ \\
Etanol/HCl 1,5N (85: $15 \mathrm{v} / \mathrm{v})$ & $15,22^{\mathrm{Bd}} \pm 0,11$ & $127,22^{\mathrm{Ab}} \pm 0,42$ \\
Água/ $\mathrm{HCl} 1,5 \mathrm{~N}(85: 15 \mathrm{v} / \mathrm{v})$ & $15,82^{\mathrm{Bbc}} \pm 0,09$ & $121,29^{\mathrm{Ac}} \pm 0,02$ \\
Etanol/água $(70: 30 \mathrm{v} / \mathrm{v})$ & $12,04^{\mathrm{Bf}} \pm 0,05$ & $112,17^{\mathrm{Ad}} \pm 0,23$ \\
Metanol/água $(70: 30 \mathrm{v} / \mathrm{v})$ & $16,14^{\mathrm{Bb}} \pm 0,06$ & $137,10^{\mathrm{Aa}} \pm 0,11$ \\
Etanol $(99,9 \%)$ & $14,24^{\mathrm{Be}} \pm 0,09$ & $41,69^{\mathrm{Ag}} \pm 0,23$ \\
Metanol $(99,9 \%)$ & $16,06^{\mathrm{Bb}} \pm 0,29$ & $62,27^{\mathrm{Ae}} \pm 0,00$ \\
\hline
\end{tabular}

Letras maiúsculas diferentes na mesma linha indicam diferença significativa entre os tipos de polpa pelo teste tStudent $(\mathrm{p}<0,05)$. Letras minúsculas na mesma coluna indicam diferença estatisticamente significativa entre os tipos de solvente, pelo teste de Tukey $(\mathrm{p}<0,05)$. Fonte: Autores.

O solvente mais eficiente para a extração das antocianinas tanto da polpa congelada quanto da liofilizada, foi o metanol acidificado, seguido pelo solvente hidrometanólico. A água e o etanol puro foram os menos eficazes para essa extração. Borges et al. (2011) avaliaram diferentes tipos de solventes na extração de antocianinas da polpa de jussara (Euterpe edulis) e verificaram que o metanol/ $\mathrm{HCl}(1,5 \mathrm{M})$ e o etanol/ $\mathrm{HCl}(1,5 \mathrm{M})$ apresentaram-se mais eficientes quando comparados a combinações de outros solventes como água/ $\mathrm{HCl}(0,01 \mathrm{M})$ ou solventes isolados como o metanol e/ou etanol puro. A maior eficiência do metanol acidificado na extração de antocianinas pode ser explicada pela maior estabilidade das mesmas em um solvente de maior polaridade e em pH ácido (Bennemann, et al., 2018).

A polpa liofilizada apresentou atividade antioxidante significativamente superior $\left(24,19 \mu \mathrm{mol} \mathrm{TE} \mathrm{g}^{-1}\right)$ à congelada

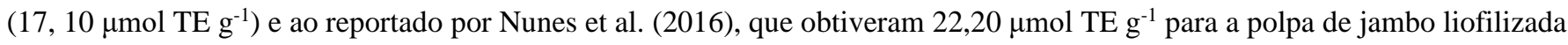
frente ao radical livre DPPH empregando Metanol/água (8:2 v/v) como solvente de extração. Correia et al. (2012) apontam que os fenólicos e as antocianinas seriam os principais responsáveis pela atividade antioxidante em resíduos de frutas tropicais em pó, o que reforça a teoria de que também tenham sido os responsáveis pela atividade antioxidante neste estudo.

Segundo Melo et al. (2011), diferenças entre variedades, região geográfica, safra, condições climáticas, peculiaridades de processamento e métodos de extração podem interferir nos resultados do teor de compostos fenólicos, bem como na expressão da atividade antioxidante de materiais vegetais. Nesta pesquisa estes fatores também impactaram os teores de flavonoides amarelos e o conteúdo total de antocianinas monoméricas da polpa de jambo vermelho.

\section{Conclusão}

O metanol acidificado $(85: 15 \mathrm{v} / \mathrm{v})$ foi o melhor solvente na extração de compostos fenólicos e de antocianinas monoméricas totais da polpa de jambo, enquanto a água pura destacou-se na extração de flavonoides amarelos. Assim, este 
estudo pode ser utilizado como referência para escolha de tipos e combinações de solventes mais apropriados para a extração de bioativos de frutos de jambo.

As polpas liofilizada e congelada obtidas sem aditivos sintéticos apresentaram características tecnológicas adequadas para industrialização e destacaram-se especialmente como fonte de compostos bioativos. Todavia, a liofilização preservou melhor a estabilidade dos compostos bioativos estudados, sugerindo-se esse método como o mais apropriado para obtenção da polpa de jambo, a fim de preservar melhor o potencial funcional deste fruto.

Sugere-se para pesquisas futuras determinar a atividade antioxidante da polpa de jambo vermelho proveniente do Estado do Rio Grande do Norte-RN pelos métodos FRAP e ABTS; identificar o perfil fitoquímico dos compostos bioativos e avaliar o potencial tecnológico do fruto na obtenção de produtos lácteos fermentados.

\section{Referências}

AOAC Association Official Analytical Chemists. (2000). Official Methods of Analysis. (12a ed.).

Augusta, I. M., Resende, J. M., Borges, S. V., Maia, M. C. A., \& Couto, M. A. P. G. (2010). Caracterização física e química da casca e polpa de jambo vermelho (Syzygium malaccensis, (L) Merryl \& Perry). Ciência e Tecnologia de Alimentos, 30(4), 928-932. https://doi.org/10.1590/S010120612010000400014.

Batista, A. G., Silva, J. K., Cazarin C. B. B., Biasoto., Sawaya, A. C. H. F.,Prado, M. A., \& Maróstica Júnior, M. R. (2016). Red-jambo (Syzygium malaccense): Bioactive compounds in fruits and leaves. LWT - Food Science and Technology, 76, $284-291$.

https://doi.org/10.1016/j.lwt.2016.05.013

Bennemann, G. D., Botelho, R. V., Torres, Y. R., Camargo, L. A., Khalil, N. M., Oldoni, T. L. C., \& Silva, D. H. (2018). Compostos bioativos e atividade antirradicalar em farinhas de bagaço de uvas de diferentes cultivares desidratadas em liofilizador e em estufa. Brazilian Journal of Food Technology, 21, 1-10. https://doi.org/10.1590/1981-6723.20517

Borges, G. S., Vieira, F. G. K., Copetti, C., Gonzaga, L. V., \& Fett, R. (2011).Optimization of the extraction of flavanols and anthocyanins from the fruit pulp of Euterpe edulis using the response surface methodology. Food Research International, 44(3), 708-715. https://doi.org/10.1016/j.foodres.2010.12.025

Brand-Williams, W., Cuvelier, M. E., \& Berset, C. (1995). Use of a free radical method to evaluate antioxidant activity. LWT-Food Science and Technolology 28(1), 25-30. https://doi.org/10.1016/S0023-6438(95)80008-5

Cano-Chauca, M., Stringheta, P. C., Ramos, A. M., \& Cal-Vidal, J. (2005). Effect of carriers on the microstructure of mango poder spray drying and its functional characterization. Innovative Food Science and Technology, 6(4), 420-428. https://doi.org/10.1016/j.ifset.2005.05.003

Carvalho, A. V., Mattietto, R. A., \& Beckman, J. C. (2017). Estudo da estabilidade de polpas de frutas tropicais mistas congeladas utilizadas na formulação de bebidas. Brazilian Journal of Food Technology, 20, 1-9. http://dx.doi.org/10.1590/1981-6723.2316

Correia, R. T. P., Borges, K. C., Medeiros, M. F., \& Genovese, M. I. (2012). Bioactive compounds and phenolic-linked functionality of powdered tropical fruit residues. Food Science Technology International, 18(16), 539-547. 10.1177/1082013211433077

Francis, F. J. (1982). Analvsis of anthocyanins. In: Markakis, P. Anthocyanins as food colors. Academic Press.

Giusti, M., \& Wrolstad, R. E. (2001). Characterization and Measurement of Anthocyanins by UV-Visible Spectroscopy. Current Protocols in Food Analytical Chemistry; S1-13.

Gordon, A., Jungfer, E., Silva, B. A., Maia, J. G. S., \& Marx, A. (2011). Phenolic constituents and antioxidant capacity of four underutilized fruits from the amazon region. Journal of Agricultural and Food Chemistry, 59(14), 7688-7699.

https://doi.org/10.1021/jf201039r

Li, G., Zhang, Y., Wu, P., Chen, N., Wu, Z., Yang, L., Qiu, R., Wang, G., \& Li, W. (2015). New phloroglucinol derivatives from the fruit tree Syzyygium jambos and their cytotoxic and antioxidant activities. Journal of Agricultural and Food Chemistry, 64 (1), $10257-10262$.

https://doi.org/10.1021/acs.jafc.5b04293

Lichtenthaler, H. K., \& Buschmann, C. (2001). Chlorophylls and carotenoids: Measurement and characterization by UV-VIS spectroscopy. Current Protocols in Food Analytical Chemistry; S1-8.

López-Córdoba, A., Deladino, L., Agudelo-Mesa, L., \& Martino, M. (2014). Yerba mate antioxidant powders obtained by co-crystallization: Stability during storage. Journal of Food Engineering, 124, 158-165. https://doi.org/10.1016/j.jfoodeng.2013.10.010

Melo, P. S., Bergamaschi, K. B., Tiveron, A. P., Massarioli, A. P., Oldoni, T. L. C., Zanus, M. C., Pereira, G. E., \& Alencar, S. M. (2011). Composição fenólica e atividade antioxidante de resíduos agroindustriais. Ciência Rural, 41(6), 1088-1093. https://doi.org/10.1590/S0103-84782011000600027

Milani, L. I. G., Terra, N. N., Fries, L. L. M, Cichoski, A. J., Rezer, A. P. S.,Backers, A. M., \& Parodia, C. G. (2012). Atividade antioxidante e antimicrobiana in vitro de extratos de caqui (Diospyros kaki L.) cultivar Rama Forte. Brazilian Journal of Food Technology, 15(2), 118-124. https://doi.org/10.1590/S198167232012005000003 
Moura Filho, J. M., Nagai, L. Y., Nascimento, L. C. S., Cavalcante Neto, A. A., \& Penna, A. L. B. (2017). Determinação do solvente ótimo para extração dos compostos fenólicos do fruto de buriti. Brazilian Journal of Food Research, 8(3), 22-28.

Retirado de: https://periodicos.utfpr.edu.br/rebrapa/article/view/3992/pdf

Nellis, S. C., Correia, A. F. K., \& Spoto, M. H. F. (2017). Extração e quantificação de carotenoides em minitomate desidratado (Sweet Grape) através da aplicação de diferentes solventes. Brazilian Journal of Food Technology, 20, 1-5.

https://doi.org/10.1590/1981-6723.15616

Nunes, P. C., Aquino, J. S., Rockenbach, I., \& Stamford, T. L. M. (2016). Physico-chemical characterization, bioactive compounds and antioxidant activity of malay apple [Syzygium malaccense (L.) Merr. \& L.M. Perry]. PloS One, 11(6), 1-11. https://doi.org/10.1371/journal.pone.0158134

Van Ngo, T., Scarlett, C. J., Bowyer, M. C., Ngo, P. D., \& Vuong, Q. V. (2017). Impact of different extraction solvents on bioactive compounds and antioxidant capacity from the root of salacia chinensis. Journal of Food Quality, 2017, 1-8. https://doi.org/10.1155/2017/9305047

Obanda, M., Owuor, P. O., \& Taylor, S. J. (1997). Flavanol composition and caffeine content of green leaf as quality potential indicators of kenyan black teas. Journal of the Science of Food and Agriculture, 74(2), 209-215. https://doi.org/10.1002/(SICI)1097-0010(199706)74:2<209::AID-JSFA789>3.0.CO;2-4

Oyinloye, T. M., \& Yoon, W. B. (2020). Effect of freeze-drying on quality and grinding process of food produce: a review. Processes, 8(354), 1-23. DOI: $10.3390 /$ pr8030354

Pereira, A. S., Shitsuka, D. M., \& Shitsuka, F. J. P. R. (2018). Metodologia da pesquisa científica, UFSM.

Kibe, M. N., Konyole, S., Nguka, G., Oloo, M. O., Kathure, D., \& Wangari, P. M. (2017). The role of phytochemicals in prevention and control of chronic diseases. International Journal of Current Research, 9(12), 62540-62543. https://www.journalcra.com/sites/default/files/issue-pdf/27237.pdf

Krumreich, F., D’Avila, R. F., Freda, S. S., Chim, J. F., \& Chaves, F. C. (2016). Análises físico-químicas e estabilidade de compostos bioativos presentes em polpa de uvaia em pó obtidos por métodos de secagem e adição de maltodextrina e goma arábica. Revista Thema, 13(2), 4-17. https://doi.org/10.15536/thema.13.2016.4-17.351

Rajbhar, K., Dawda, H., \& Mukundan, U. (2015). Polyphenols: methods of extraction. Scientific Reviews \& Chemical Communications, 5(1), 1- 6. https://www.tsijournals.com/articles/polyphenols-methods-of-extraction.pdf

Rocha, B. R., Maciel, E. A., Oliveira, S. R. M., Terence, Y. S., \& Silva. B. A. (2021). Influência dos alimentos funcionais na incidência das doenças crônicas não transmissíveis (DCNT). Intercontinental Journal on Physical Education, 3(1), 1-20. http://www.ijpe.periodikos.com.br/articl e/60274ea60e8825b8147e523a/pdf/ijpe-3-1-e2020021.pdf

Santos, M. C. P., \& Gonçalves, E. C. B. A. (2016). Effect of different extracting solvents on antioxidant activity and phenolic compounds of a fruit and vegetable residue flour. Scientia Agropecuaria, 7(1), 07-14. http://dx.doi.org/10.17268/sci.agropecu.2016.01.01

Shajib, M. T. I., Kawser, M., Miah, M. N., Begum, P., Bhattacharjee, L., Hossain, A., Fomsgaard, I. S., \& Islam, S. N. (2013). Nutritional composition of minor indigenous fruits: cheapest nutritional source for the rural people of Bangladesh. Food Chemistry, 140, 466-470. 10.1016/j.foodchem.2012.11.035.

Silva-Espinoza, M. A., Ayed, C., Foster, T., Camacho, M. M., \& Martínez-Navarrete, N. (2020). The impact of freeze-drying conditions on the physicochemical properties and bioactive compounds of a freeze-dried orange puree. Foods, 9(32), 1-15. 10.3390/foods 9010032

Silva, L. M. R., Figueiredo, E. A. T., Silva, R. N. M. P., Vieira,I. G. P.,Figueiredo, R. W., \& Brasil, I. M. (2014). Quantification of bioactive compounds in pulps and by products of tropical fruits from Brazil. Food Chemistry, 143, 398-04. 10.1016/j.foodchem.2013.08.001. 